\title{
Effects Of Mixed Vaccinations Against Newcastle Disease And Infectious Bursal Disease On Immune Response, Feed Consumption And Weight Gain In Broilers.
}

\author{
Okwor, E. C., Eze, D. C., Anyanwu, M. U., Okpe, C. B., And Eze, P. C. \\ Department of Veterinary Pathology and Microbiology University of Nigeria, Nsukka
}

\begin{abstract}
The effect of mixed vaccinations against Newcastle disease (ND) and Infectious bursal disease (IBD) on immune response, feed consumption and weight gain were studied in 21 days old broilers. The trial was carried out to ascertain the safety of this practice which is intended to reduce labour, cost and stress associated with chasing and catching during individual bird vaccinations in large flocks. The two diseases are among the common viral diseases found in Nigeria; a country with an expanding poultry population that is creating major impact in its meat industry. Their prevention is largely by vaccination and vaccinations with live vaccines can precipitate reactions. A total of 120 broilers were assigned into 4 groups (G1, G2, G3 and G4) of 30 chickens each at 21 days of age. Chicken in G1 were vaccinated with ND vaccine, La Sota which was given singly at 21 days of age and repeated at 35 days of age. Chickens in G2 were vaccinated with both ND vaccine, La Sota and $I B D$ vaccine, which were given simultaneously at 21 days of age and repeated at 35 days of age. Chickens in G3 were vaccinated with both vaccines but the vaccines were given singly with $N D$ vaccine given at 21 days of age and repeated at 35 days of age while IBDV vaccine was given at 28 days of age and repeated at 42 days of age. Chickens in G4 were vaccinated with IBDV vaccine only which was given at 21 days of age and repeated at 35 days of age. Serum samples were collected from 10 chickens in each group for serology. The chickens in the groups were monitored for feed consumption and weight gain. Results showed that mixed vaccination of the chicken was well tolerated. Mixed vaccination did not affect immune response of the chickens. There was no significant difference $(p>0.05)$ in both feed consumption and weight gain among chickens in the different groups.
\end{abstract}

\section{Introduction}

Newcastle disease (ND) is an acute, highly contagious and rapidly spreading viral disease affecting birds of all ages with clinical signs and lesions that vary in severity and also with the species of birds affected (Alexander, 2000; Abdu et al., 2004; and Saidu et al., 2006). The virus, Newcastle disease virus (NDV) is a single stranded, negative sense RNA virus classified in the genus Avulavirus and in the family Paramyxoviridae (Mayo, 2002). Infectious bursal disease (IBD) is a highly contagious viral disease of young chickens caused by a virus, Infectious bursal disease virus (IBDV), which is a member of the genus, Avibirnavirus of the family Birnaviridae (Delmas et al., 2004). The virus is a double stranded RNA virus that has bi-segmented genome (Wu et al., 2007; Zhu et al., 2008). Both diseases rank high among the viral infections of poultry in Nigeria. According to Moges (2010), ND is the most prevalent and economically important disease problem affecting birds in developing countries. Infectious bursal disease is the second most important and economically prevalent viral disease of chickens in Nigeria.

Poultry products are among the fastest growing components of global agricultural demands. Nigeria like other developing countries is experiencing rapid growth in its poultry sector. This is being driven by rising incomes and the expanding middle class, together with the fact that poultry products are among the cheapest sources of protein. At the same time, the market and production context of poultry production has been changing rapidly. Rapid economic growth and urbanization in developing countries has resulted in fast expansion of industrial large scale, vertically integrated, poultry production units with the resultant large scale commercial poultry farming (Adeyemo and Onikoyi, 2012). However, this rapid growth and expansion still have some factors militating against it. One of the major factors in Nigeria is infectious diseases especially the two mentioned above.

Vaccination still remains one of the best approaches in the control of viral diseases of poultry in Nigeria. This has helped to reduce the incidence of these diseases, thereby reducing economic losses and increased production output with resultant gain to farmers. In developing countries these vaccines are available most often as live vaccines. The use of live vaccines can result in vaccination reactions especially if the birds are stressed (Alexander, 2003). With the ever expanding flock sizes and the increasing farm size it is possible for individual bird inoculation, which ensures even distribution of the vaccine but involves the chasing and catching of the birds for hours, to constitute a major stress. It also increases labour and cost. To save labour, and possibly 
minimize stress due to chasing and catching of the birds, it may be possible to administer the two vaccines simultaneously. However, this procedure may have some effects on immune responses to the vaccines, feed consumption and weight gain. This work investigated the effect of simultaneous vaccinations against ND and IBD on immune responses to NDV, feed consumption and weight gain in broilers using live attenuated vaccines. Qualitative immune responses to IBD were also assessed. The result will serve as a guide in formulating a vaccination programme involving simultaneous use of both vaccines. This will be helpful in reducing labour and stress associated with individual application of vaccines in poultry. It will also be of help in emergency vaccinations when the birds are exposed or at risk of being infected.

\section{Materials And Methods}

Experimental Chickens: A total of one hundred and twenty (120) broilers were used in the study. The chickens were sourced at day old from a commercial hatchery in Nigeria and housed at the animal house of the Department of Veterinary Pathology and Microbiology, University of Nigeria, Nsukka. The vaccination history of the parent stock was not available and the birds were not vaccinated in the hatchery. The birds were reared on deep litter with feed and water given ad-libitum. Anti-stress vitamins and coccidiostats were given at strategic periods.

At 21 days of age, the birds were randomly assigned into 4 groups (G1, G2, G3 and G4) of 30 birds each. Chicken in G1 were vaccinated with ND vaccine, La Sota which was given singly at 21 days of age and repeated at 35 days of age. Chickens in G2 were vaccinated with both ND vaccine, La Sota and IBD vaccine, Intermediate Strain which were given simultaneously at 21 days of age and repeated at 35 days of age. Those in G3 were vaccinated with both vaccines but the vaccines were given singly with ND vaccine given at 21 days of age and repeated at 35 days of age while IBDV vaccine was given at 28 days of age and repeated at 42 days of age. Birds in G4 were vaccinated with IBDV vaccine only which was given at 21 days of age and repeated at 35 days of age.

Serum samples were collected from 10 birds in each group on day 21 of age and thereafter on a weekly basis for serology. Moreover, the average feed intake and the average weight gain of the birds in each group were accessed.

Serology: Collection of serum sample for serology was done through the jugular vein using $5 \mathrm{ml}$ syringes and 22 gauge x 1.5 inches needle. Two millilitres of blood was collected and transferred into vials containing no anticoagulant and kept in a slanted position for 25 minutes to allow the blood to clot. The blood was stored in the refrigerator overnight for the clot to retract and the serum released. The serum was harvested in another vial and stored at $-20^{\circ} \mathrm{C}$ until used. The serum was used for Haemagglutination inhibition (HI) test for the detection of ND antibodies (Beard, 1989) and Indirect Haemagglutination (IHA) test for the detection of IBDV antibodies (Hussain et al., 2003).

HI test: This was carried out as described by Beard (1989). Four Haemagglutinating units (4HU) of the NDV antigen were used. Briefly, a serial two-fold dilution of the serum sample was made with Phosphate Buffered Saline (PBS) in microtitre plate from well number one to well number twelve. Equal volume of the viral antigen was then added to each of the wells. The plate was left at room temperature so as to provide ample time for antigen- antibody reaction. After this, equal volume of the already prepared $0.5 \%$ chick erythrocytes were added to all the wells and allowed to stand at room temperature for up to 40 minutes. The HI titre was read as the highest dilution of serum causing complete inhibition of agglutination. Therefore, positive results showed inhibition of agglutination of erythrocytes with characteristic settling of the cell (smooth button) while negative result showed agglutination of erythrocytes with dispersion and rough settling of the cells (no button).

IHA test: This was carried out as described by Hussain et al (2013). Briefly, 1\% human O+ erythrocytes were prepared and sensitised with IBD antigen (virus). Two fold serial dilution of the serum sample was made in PBS in a row of micropipette. After this, equal volumes of the sensitized erythrocytes were added to each well, the plate tapped gently to ensure even dispersion of the erythrocytes and then incubated at $37^{\circ} \mathrm{C}$ for about 45 minutes. After, incubation, the plates were read and the reciprocal of the last dilution showing complete agglutination of the erythrocytes was recorded as the IHA titre of the serum sample.

Feed Intake: The daily feed intakes in all the groups were monitored. A measured amount of feed per group was distributed in their feed troughs and the birds allowed feed for 24 hours. The remaining feed after this period was weighed and replaced with another weighed amount and this was repeated on a daily basis for the period of the experiment. The difference between the quantity given and the remainder was calculated as the daily feed intake. The average feed intake per bird per week was calculated for each group.

Weight Gain: The weight gains of the birds in all the groups were also monitored. This was done on a weekly basis. Ten birds in a group were randomly selected and weighed and the average weight calculated. The difference between the calculated average weight and that calculated the previous week for the group was recorded as the weight gain for that group at the end of the particular week. 
Statistical analysis: The data obtained were analysed statistically using the one-way analysis of variance (ANOVA).

\section{Results}

Immune response: Figure 1 shows the geometric mean HI antibody titres against NDV in the four groups as estimated at different periods during the experiment. In G1, G2 and G3, the titres declined immediately after vaccination at 21 days of age but increased later reaching peak at approximately 14 days post vaccination (PV). With booster vaccination in the three groups at 35 days of age the titres rose significantly $(p<0.05)$ reaching peak again at 14 days PV. Chickens in G4 showed a steady decline in antibody levels which became undetectable by 35 days of age. Chickens in G2 and G3 which received the two vaccines simultaneously and separately respectively did not show significant differences $(p>0.05)$ in their mean $\mathrm{HI}$ antibody titres on days 7 , 14, 21, 28 and 35 PV. However, the increase in antibody was higher in G2 and G3 when compared with G1. Moreover, the antibody response in G2 was numerically higher in G2 than in G3. The IHA showed good immune response to IBD vaccination in G2, G3, and G4. It declined and was undetectable by day 35 in G1.

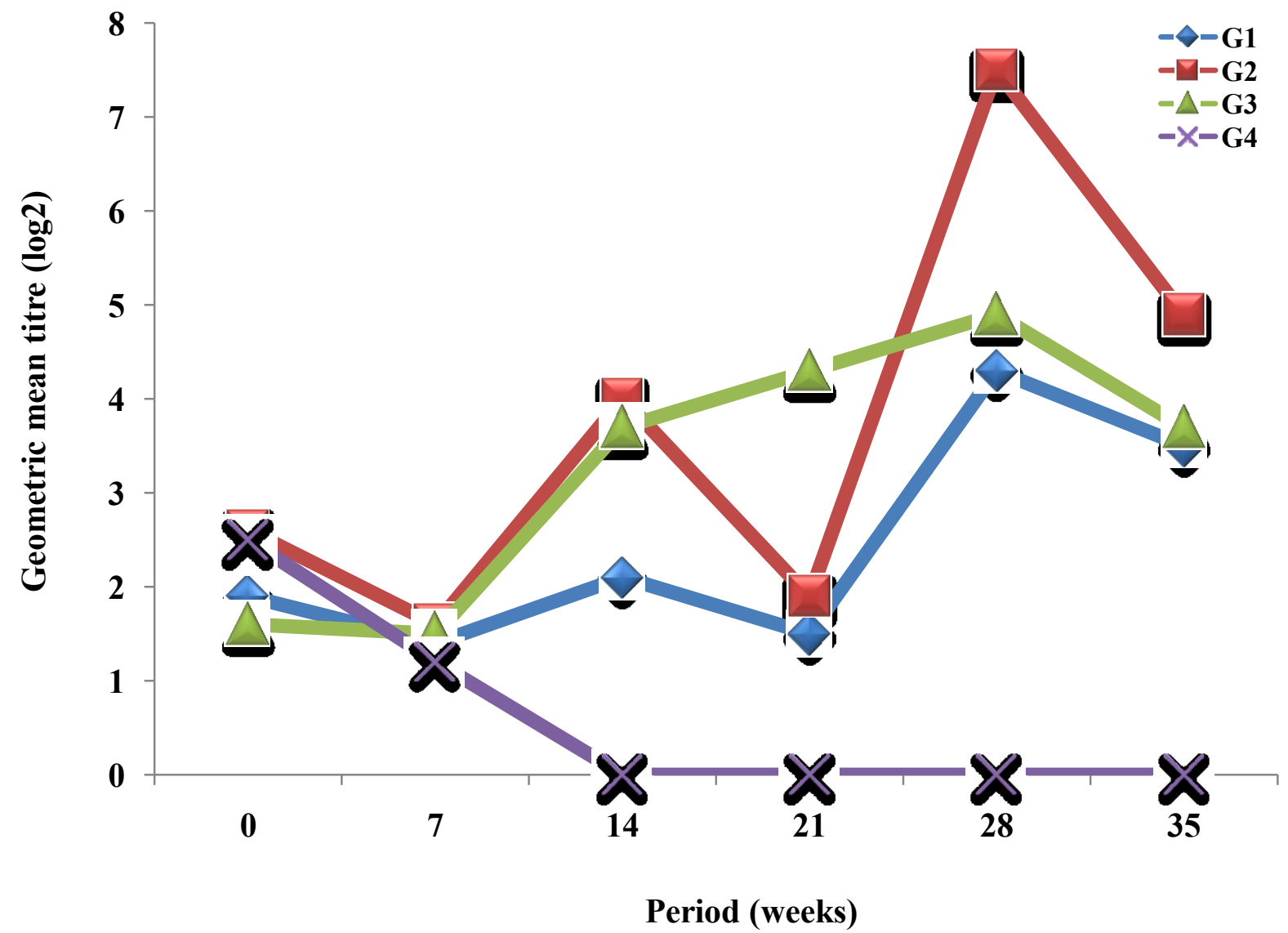

Figure 1: Graph showing the effect of simultaneous vaccinations against ND and IBD on immune responses to NDV in broilers.

Feed intake: The feed consumption among chickens in the four groups did not vary significantly $(p>0.05)$ throughout the experiment (Figure 2). It showed a weekly steady increase in the quantity of feed consumed by the chicken in all the groups. 


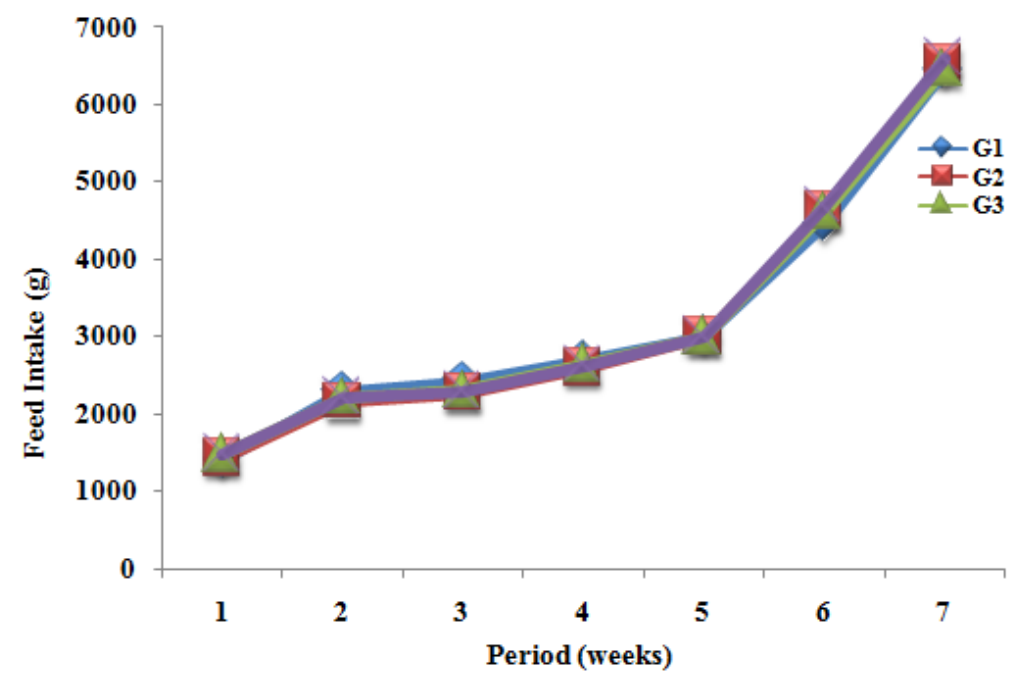

Figure 2: Graph showing the effect of simultaneous vaccinations against ND and IBD on, feed consumption in broilers.

Weight gain: The mean weight of chickens in the 4 groups differed significantly $(\mathrm{p}<0.05)$ at 21 days of age which was the day they were assigned into groups. However, the weight gain in chickens did not differ significantly $(\mathrm{p}<0.05)$ among chickens in the 4 groups throughout the period of the experiment. The differences in body weight among the groups narrowed down and towards the end of the experiment, were not statistically significant $(\mathrm{p}<0.05)$ (Figure 3 ).

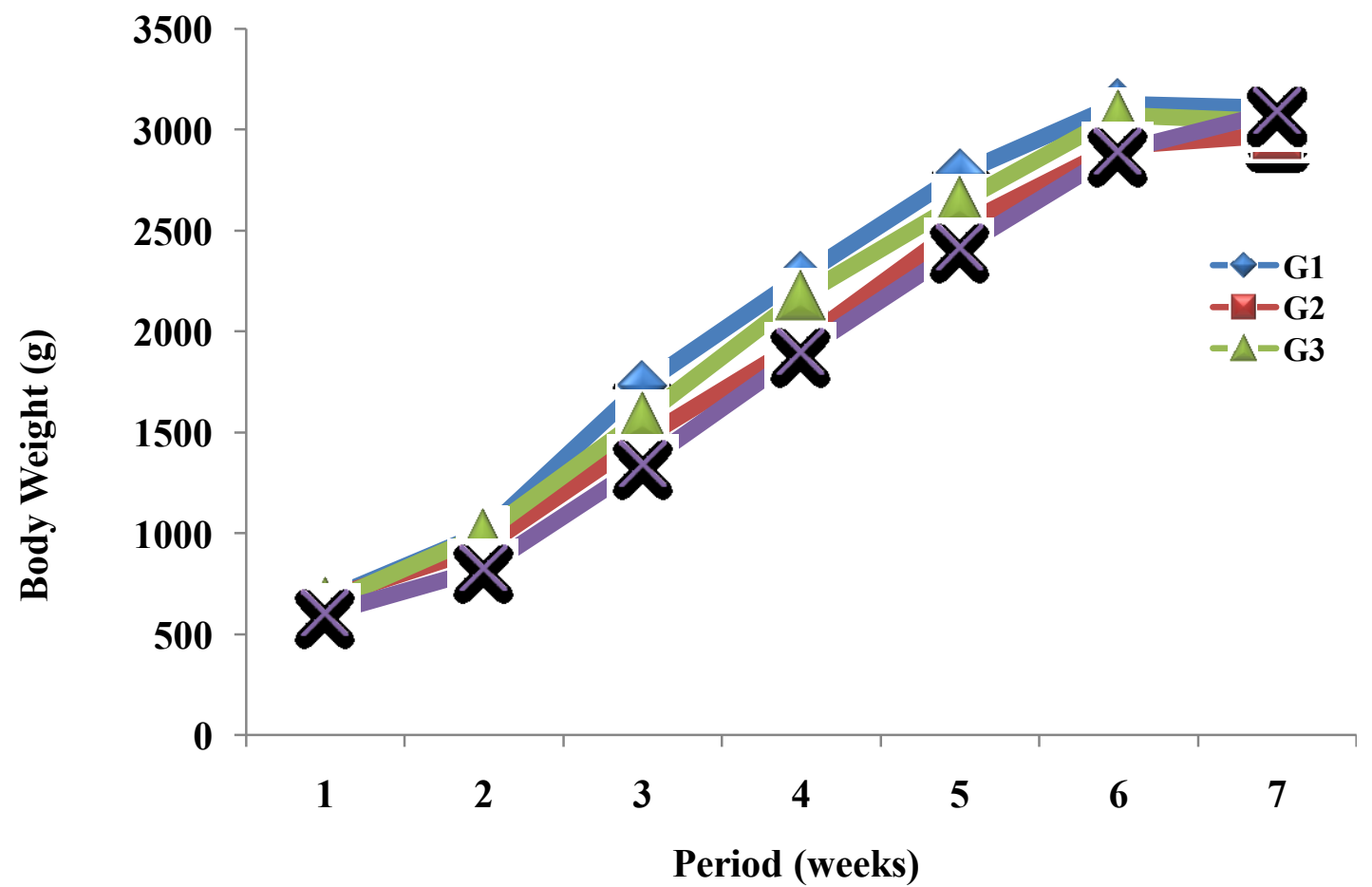

Figure 3: Graph showing the effect of simultaneous vaccinations against ND and IBD on weight gain in broilers

\section{Discussions}

The results obtained in this study showed that simultaneous vaccination of broilers against Newcastle disease and Infectious bursal disease was well tolerated. The mixed vaccination did not reduce the level of immune response, and did not affect feed intake and weight gain. The difference in weight as observed at 21 days of age in the groups was not due to group treatments as the chickens were assigned into groups on this day. It was most likely due to sampling as heavier birds may have been picked for weighing by chance. As seen in the subsequent weeks there was no difference in weight gain among the groups especially at the end of the experiment. 
Reports in literature concerning mixed vaccinations in chickens vary. Our findings are similar to that previously reported by many investigators. For instance, Cholakova (1985) working on the effects of associated vaccination of poultry against some diseases found that the simultaneous application of live vaccines against infectious bronchitis, Newcastle disease, and infectious bursitis in broilers, pullets, and parent stock was well tolerated with no harmful after effects whatever. He concluded that the immunity built up with the simultaneous use of the three vaccines was not inferior in effectiveness to that conferred with the use of two vaccines or only one of them. Zamani Moghaddam et al. (2007) studied the effect of mixed applications of live vaccines of Newcastle disease and Infectious bronchitis in chickens and found no effect on weight gain and feed conversion ratio. They also found no difference in protection in chickens when the vaccines were mixed and when given singly. Chansiripornchai and Sasipreeyajan (2006) though working on NDV vaccines found that simultaneous vaccinations of broilers with Hitchner B1 or Ulster 2C and inactivated oil adjuvant vaccine gave good protection even without a booster vaccination. The body weight gain, feed intake and feed conversion ratio were not affected in each case. Conversely, Ali et al (2004) found some effects on immune responses when live Newcastle disease and Infectious bursal disease vaccines were used to vaccinate chickens. They found adverse effect on immune response to ND vaccine, La Sota when used after live IBD vaccine. Similarly, Thornton and Muskett (1973) in their study indicated that protection of chickens against challenge of virulent NDV is higher in those chickens received B1 vaccine alone, as compared to those received B1 and IBD vaccine.

Vaccination of chickens is practiced to provide some degree of protection against disease. The antigens stimulate the bird's immune system which responds more effectively in controlling losses if there is infection. In order to do this the vaccine virus must infect target cells and replicate to increase their numbers to effectively stimulate the immune system. This may result in a vaccine reaction. Questions arise as to vaccine reaction using live vaccines as it has to do with production parameters and therefore cost. Performances of chickens that can be influenced by vaccination are feed conversion, body weight gain, uniformity, and seroconversion (Butcher et al., 2011) and these parameters are very important especially in broilers. Losses associated with decreased body weight and uniformity, poor feed conversion and increased condemnation will affect profit associated with broiler production. Therefore vaccination programme that will reduce labour and cost should also not affect immune response and performance of the birds. Porchezhian and Punniamurthy (2006) considered broiler production to be an economical enterprise only when broilers convert efficiently what they eat and noted that prophylactic measures used in the control of disease should exert little or no effect on broiler production parameters. Therefore our investigation showed no effect on the production parameters when healthy chickens were vaccinated simultaneously against ND and IBD using La Sota and Intermediate strain respectively.

As noted before, there is a considerable variation in response of birds to mixed vaccines. An important observation in this study was that the used of ND and IBDV vaccines in G2 and G3 gave higher immune response to ND than when only ND vaccine was used in G1. Moreover, the immune response to ND was again higher with the simultaneous use of the two vaccines in G2 when compared with the single use of both vaccines at different periods in G3. The above observations were similar to that reported by some workers on mixed vaccinations in poultry. Cardoso et al. (2006) reported that the utilization of polyvalent vaccinal programs have a different efficacy comparing to monovalent vaccinations when Newcastle disease, avian infectious bronchitis, and infectious bursal disease vaccinations are applied. This way, the use of vaccinations to infectious bursal disease in polyvalent vaccinal programs is desirable due to improvement of NDV response and reduction of possible interference of IBV under NDV. Cholakova (1985) reported that combination of two or three live vaccines against Infectious bursal disease, Newcastle disease and Infectious bronchitis were safe and effective on the basis of the humoral immune responses after 20 or 30 days. Zhamani Moghaddam et al. (2007) found numerically higher antibody titres in the group of chickens that had mixed vaccination with ND and IB when compared the group that received IB vaccine alone. The authors argued that this higher immune response in mixed vaccination might be due to the increased histopathologic lesions in the tissues of the treatment groups, which may assist to increased immune response due to increased immune tissue stimulation. Nakamura et al. (1992) reported that administration of mixed vaccine has demonstrated to induce deciliation, hyperplasia, hyperaemia and some lesions in the tracheal epithelial mucosa. The reason for the higher immune response to ND with the simultaneous use of the two vaccines in G2 when compared with the single use of both vaccines at different periods in G3 can be explained by the observations of Ali et al. (2004) who observed that vaccination of chicks with ND vaccine containing La Sota strain of the virus was adversely affected by live IBD vaccine when administered first. They advocated that chickens be first vaccinated against ND before vaccination against IBD. Some strains of IBD used in live vaccines can induce bursal atrophy and immunosuppression in chickens (Lukert and Mazariegos, 1985; Boudaoud and Alloui, 2008). This meant that the simultaneous use of both vaccines in G2 did not allow time for destruction of some of the lymphocytes by IBD vaccine before immune response to ND. 
The need to reduce cost, labour and stress associated with individual chasing and catching of birds (which assures uniform vaccine take) during vaccination in large scale poultry production has also lead to the combination of poultry vaccines against multiples diseases into single dose. Recombinant NDV (La Sota strain) expressing VP2 protein of IBDV have also been produced and found to generate antibody response against both ND and IBD and conferred complete protection against both viruses especially after booster immunisation (Huang et al., 2004).

Conclusively, our investigation have shown that mixed vaccination against ND and IBD using live vaccines did not affect immune response, feed intake and weight gain in healthy broilers. Moreover, the findings showed numerically higher immune response in the groups that received both vaccines especially when they were given simultaneously than in the group that received ND vaccine alone. Therefore in order to reduce labour, cost and stress in large scale individual bird vaccinations which guarantees even distribution of the vaccine, simultaneous application of ND and IBD vaccines is advocated. This however will be restricted to healthy birds and also to the lentogenic strains of ND vaccine and intermediate strain of IBD vaccine.

\section{References}

[1]. Abdu, P. A., Manchang, T. K., and Saidu, L. (2004). The epidemiology and clinicopathological manifestations of Newcastle disease virus in Nigerian local chickens. Proceedings of the $41^{\text {st }}$ Congress of the Nigerian Medical Association. NVRI Vom. p 57.

[2]. Adeyemo, A. A., Onikoyi, M. P. (2012). Prospects and challenges of large scale commercial poultry production in Nigeria. Agricultural Journal. 7: 388 - 393.

[3]. Alexander, D. J. (2000). Newcastle disease and other avian Paramyxoviruses. Revue Scientifique et Technique de l'Office International des Epizooties. 19: 443 - 462

[4]. Alexander, D. J. (2003). Newcastle disease and other avian paramyxovirus and pnuemovirus infections. In: Y. M. Saif (ed). Diseases of Poultry. $11^{\text {th }}$ Edition. Iowa State University Press. USA. Pp $64-87$.

[5]. Ali, A. S., Abdalla, M. O., and Mohammed M. E. H. (2004). Interaction between Newcastle disease and Infectious bursal disease vaccines commonly used in Sudan. International Journal of Poultry Science. 3 (4): 300 - 304.

[6]. Boudaoud, A. and Alloui, N. (2008). Evaluation of the safety of live attenuated vaccine viruses against Infectious bursal disease (Gumboro disease) in conventional broiler chicks. Rev. Sci. Tech. 27(3) $793-802$.

[7]. Butcher, G. D., Miles, R. D., and Nilipour, A. H. (2011). Newcastle and Infectious bronchitis vaccine reaction in commercial broilers. Veterinary Medicine. Institute of Food and Agricultural Sciences. University of Florida. Pp $1-4$.

[8]. Cardoso, W. M., Aguiar Filho, J. L. C., Romao, J. M., Salles, R. P. R., Camara, S. R., Siqueira, A. A., Oliveira, W. F., Sobral, M. H.N. R., and Texeira, R. S. C. (2006). Interference of Infectious bursal disease virus on antibody production against Newcastle disease and Infectious bronchitis virus. Brazilian Journal of Poultry Science. 8(3) $177-182$.

[9]. Chansiripornchai, N. and Sasipreeyajan, J. (2006). Efficacy of live B1 or Ulster 2C Newcastle disease vaccine simultaneously vaccinated with inactivated adjuvant vaccine for protection of Newcastle disease vaccine in broiler chickens. Acta Veterinaria Scandinavia. $48: 2-6$.

[10]. Cholakova, R. (1985). Associated vaccination of poultry against Infectious bronchitis, Newcastle disease and Infectious bursitis. Veterinarno Meditsinski Nauki, 22 (9) $32-38$.

[11]. Delmas, B., Kibenge, F. S. B., Leong, J. C., Mundt, E., Vakharia, V.N. (2004) Birnaviridae. In: Fauquet, C. M., Mayo, M. A., Maniloff, J. (editors). Virus Taxonomy Eighth Report of the International Committee on Taxonomy of Viruses. U Desselberger \& LA Ball London: Academic Press. pp. 561-569.

[12]. Huang, Z., Elankumaran, S., Yunus, A. S., and Samal, S. K. (2004). A recombinant Newcastle disease virus (NDV) expressing VP2 protein of Infectious bursal disease virus (IBDV) protects against NDV and IBDV. Journal of Virology. 78(18): 10054 10063.

[13]. Hussain, I., Zahoor, M. A., Rasool, M. H., Mahmood, M. S., Mansoor, M. K. and Riaz, M. N. (2003). Detection of serum antibody levels against Infectious bursal disease (IBD) virus using Indirect Haemagglutination (IHA) test in commercial broilers. International Journal of Poultry Science. 2: $442-445$.

[14]. Lukert, P. D. and Mazariegos, L. A. (1985). Virulence and immunosuppressive potential of intermediate vaccine strains of infectious bursal disease virus. Journal of American Veterinary Medical Association. 187: 306 - 312.

[15]. Mayo, M. A. (2002). A summary of taxonomic changes recently approved by ICTV. Archive Virology. 147(8): 1655-1656.

[16]. Moges, F., Abera, M. and Tadelle, D. (2010): Assessment of village chicken production system and evaluation of the productive and reproductive performance of local chicken ecotype in Bure district, North West Ethiopia. African Journal of Agricultural Research, 5 (13):1739-1748.

[17]. Nakamura, K., Narita, M., Imai, K., Matsumura,T., Maeda, M., and Tanimura, T. (1992). The effect of mixed live vaccines of Newcastle disease and infectious bronchitis on the chicken respiratory tract. Journal of Comparative Pathology. $106: 341$ - 350.

[18]. Porchezhian, T. and Punniamurthy, N. (2006). Effect of oral Levamisole hydrochloride on feed intake and body weight of broiler chicks. Journal of Animal and Veterinary Advances 5(10): 847 - 848.

[19]. Saidu, L., Abdu, P.A., Tekdek, L. B., Umoh, J. U., Usman, M., and Oladele, S. B. (2006). Newcastle disease in Nigeria. Nigerian Veterinary Journal. 2: $23-32$.

[20]. Thornton, D.H. and Muskett, J.C. (1973). Comparison of immunity to Newcastledisease after vaccination with Newcastledisease vaccine given alone or together withinfections bronchitis vaccine. Veterinary Record. 92:373-374.

[21]. Wu C. C., Rubinella, P., and Lin, T. L. (2007). Molecular detection and differentiation of infectious bursal disease virus. Avian Diseases. 51(2): $515-526$.

[22]. Zamani Moghaddam, A. K. Mohammadopour, A. A. and Khajali, F. (2007). The effect of mixed live vaccines of Newcastle disease and Infectious bronchitis on systemic and local antibody responses in chickens. Iranian Journal of Veterinary Research. 8 (18): 71 -75 .

[23]. Zhu, L. O., Wu, S. L., Zhang, G. P., and Zhu, G. Q. (2008). The cellular receptor for infectious bursal disease. African Journal of Biotechnology. 7(25): 4832 - 4835. 